\title{
Social policy and law regarding education and equal social integration of persons with disabilities: model and lessons learned
}

Tavee Cheausuwantavee

Ratchasuda College, Mahidol University, Nakhon Pathom 73170, Thailand. tavee126@hotmail.com

Background: Little is known about the overview of legal essences and their enforcement regarding educational provisions for persons with disabilities (PWDs) in Thailand.

Objectives: It aims to 1) comprehensively review, establish and launch model regarding reformation of policy and law, 2) identify results of law enforcement as a past decade, present as a lesson learned on educational provisions for PWDs through consciousness raising of stakeholders.

Methods: This research is participation action research (PAR) and content analysis. As PAR, the two research sites with 13 and 15 participants as private and public education centres for PWDs respectively were voluntarily and purposively selected. Those participants included teachers, administrators, parents of PWDs. As content analysis, the results of two previous research projects were collected. Those data were analyzed by interpretation and analytic induction.

Findings: Even the rights of PWDs were more concerned by society than the past decade, but educational provisions for PWDs have still challenged and should be mainly promoted on 1) positive attitudes toward PWDs, 2) particular skills and knowledge of teachers as IEP, inclusive education and transition services 3) reasonable accommodation and assistive technologies, 4) human resource development. As PAR, inclusive education was mainly concerned by participants. It reflected what participants coped with and how they solved comprehensively.

Discussion: Problems and ineffective law enforcement on educational provisions for PWDs in Thailand existing like previous studies in other developed and developing countries. Positive attitudes of society toward PWDs, collaboration, consciousness raising and patience among stakeholders are the key success of educational provisions for PWDs in which can be enhanced through PAR.

Conclusion: The majority of law enforcement and educational equality of PWDs were still ineffective and discriminated. Thus, role of parents and private centers as well as positive attitudes and collaboration among stakeholders need to be systematically promoted. 